\title{
Reliability Sensitivity Analysis of Vehicle Components and Architecture Optimization
}

\author{
Zhe Liu, Fenghe Tao, and Changzhi Jia
}

\begin{abstract}
In the reliability designing procedure of the vehicle components, when the distribution styles of the random variables are unknown or non-normal distribution, the result evaluated contains great error or even is wrong if the reliability value $R$ is larger than 1 by using the existent method, in which case the formula is necessary to be revised. This is obviously inconvenient for programming. Combined reliability-based optimization theory, robust designing method and reliability based sensitivity analysis, numerical method for reliability sensitivity design were presented based on fourth moment technique, and the reliability sensitivity of the capstan and drive shaft in track-laying vehicle with non-normal random parameters were extensively discussed. The variation regularities of reliability sensitivity were obtained and the effects of design parameters on reliability of the capstan and drive shaft in track-laying vehicle were studied. The method presented in this paper provides the theoretic basis for the reliability design of tracked vehicle components, utilizing the reliability-based optimization and robust design method in the reliability designing procedure reduces the manufacture cost and provides the theoretical basis for the reliability and robust design of the vehicle components, the method can be very useful if it extended to correlated area of machine sensitivity design.
\end{abstract}

Index Terms-Capstan, drive shaft, reliability sensitivity design, arbitrary distribution parameters, fourth-moment technique.

\section{INTRODUCTION}

Reliability analysis of tracked vehicle components is highly related to the quality of product in terms of its service lifetime, maintenance cost, and the risk of failure. In engineering practice, given the probability distribution of basic modeling variables, reliability analysis determines the corresponding failure probability of the structure under consideration [1], [2]. Inversely, it is also significant to calibrate the distribution parameters of modeling variables given a predefined level of structural failure probability [3], [4]. The inverse procedure of reliability analysis is referred to the reliability-based design optimization in the paper. Since that available optimization procedure are usually formulated as the gradient-based trade-off process, sensitivities of structural failure probability (Pf) with respect to each distribution parameter, then, are vital to implement the optimization [5], [6]. In addition, reliability-based sensitivity analysis is considered to be important due to the fact that it is capable of evaluating the

Manuscript received December 23, 2015; revised January 15, 2016. This work was supported by The National Natural Science Foundation of China(No. 51175508) and The National Natural Science Foundation of Hebei(No. E2012506009).

Zhe Liu, Fenghe Tao, and Changzhi Jia are with the First Department, Mechanical Engineering College, PR China (e-mail: Lzlz1313@126.com). impact of design parameter changes on component safety.

Robust design theory can produce optimum results that are not sensitive to the perturbation of basic parameters [7]. The earliest idea of robust-Taugchi method [8], was originated from the field of quality control in 1960's. After introduced into the engineering world, the concept of Taugchi method can be classified into the following two categories [9]: (a)Semi-empricial or empirical robust design method, including classical Taugchi method, response surface method, and general model method, etc; (b)Robust-based optimization method, which depends on the adopted optimization method, such as the tolerance polyhedron method, gradient-based sensitivity analysis, and the method on tolerance propagation.

During the last four decades of the 20th century, there has been considerable research in the area of engineering [10]. It provided a thorough summary of researches on reliability design of vehicle components with efficient and accurate computational reliability theories. It mentioned a simple method which was presented for a second-order structural reliability approximation. The method was based on an approximating paraboloid [11], which was fitted to the limit-state surface at discrete points with minimal distance from the origin. According to the methods of reliability sensitivity considered, there are two paths [12]: one is based on the moment method, and the other is a numerical simulation on the basis of Monte-Carlo simulations.

The concept of robust design was quick spread in both academic and industry [13]-[15], since that robust design can guarantee the minimum output variance as involving a relative large noise in input variables. A reliability model and reliability-based design optimization methodology for maintenance were presented by LIU, et al. [16]. The fusion of the probabilistic finite element method(PFEM) and reliability analysis for probabilistic fracture mechanics (PFM)was presented by GESTERFIELD. And at the same time FARAVELLI introduced and discussed a stochastic finite element method [17]. It can be used for the analysis of structural and mechanical systems whose geometrical and material properties have spatial random variability. The method utilizes a polynomial expansion of the numerical nonlinear structural operator(for which actual analytical form is unknown).SUES [18] also described a method as most probable point system simulation(MPPSS) that was used to obtain system sensitivity factors, that is, the importance of each random variable to the system reliability. With the Edgeworth series, robust optimization of a coil tube-spring with non-normal distribution parameters was studied by ZHANG et al. [19]. The Edgeworth series has been defined in the infinite domain, which can be trusted to approximate any realistic structural probabilistic response defined as an 
implicit or explicit function of input random variables [20].

If certain factors on the tracked vehicle components failure have a greater impact, so in the process of design and manufacture of it needs to be strictly controlled, making it small enough to ensure that the changes in vehicle components have adequate safety and reliability; on the contrary, if the change of a factor of vehicle reliability is not significantly affected parts, vehicle components during the design and manufacturing process, can be treated as a fixed value, which can reduce the complexity of the design and manufacture. Reliability sensitivity design has been fully developed nearly forty years. However, some tracked vehicle components' parameters are non-normal distribute in fact, the reliability sensitivity analysis need to know the distributing model, so it is hard to analyze exactly the reliability of tracked vehicle components. This paper discusses the reliability-based robust design of vehicle components with non-normal distributions of parameters, based on the perturbation technique, fourth-moment technique, and the robust design method. Under the consideration of the characteristics of random variables is know, it can quickly and accurately obtain reliability sensitivity design information of vehicle components.

\section{Perturbation Method of Reliability Design}

A fundamental problem in reliability analysis is the computation of the multi-fold integral of the reliability R:

$$
R=\int_{g(\boldsymbol{X})>0} f_{X}(\boldsymbol{X}) d X
$$

where $f_{X}(\boldsymbol{X})$ denotes the probability density function of random parameter vector $\boldsymbol{X}=\left(X_{1} X_{2} \cdots X_{n}\right)^{T}$, and $g(\boldsymbol{X})$ defines the state function, representing the safe and failure states where

$$
\left\{\begin{array}{c}
g(\boldsymbol{X}) \leq 0, \text { failurestate } \\
g(\boldsymbol{X})>0, \text { safestate }
\end{array}\right.
$$

And $g(\boldsymbol{X})=0$ is the limit-state equation representing an n-dimensional surface, which may be called the "limit-state surface" or "failure surface".

The vector of random parameters $\boldsymbol{X}$ and the state function $g(\boldsymbol{X})$ are expanded as

$$
\begin{gathered}
\boldsymbol{X}=\boldsymbol{X}_{d}+\varepsilon \boldsymbol{X}_{p} \\
g(\boldsymbol{X})=g_{d}(\boldsymbol{X})+\varepsilon g_{p}(\boldsymbol{X})
\end{gathered}
$$

where $\varepsilon$ is a small parameter. The part of Eqs. (3) and (4) that is denoted by subscript $d$ is the certain part of the random parameters, and the part that is denoted by subscript $\mathrm{p}$ is the random part, having a zero mean value in the random parameters. Obviously, it is necessary for the value of the random part to be smaller than the value of the certain part. Both sides of Eqs.(3) and (4) are evaluated about the mean value of the random variables as follows:

$$
E(\boldsymbol{X})=\mathrm{E}\left(\boldsymbol{X}_{d}\right)+\varepsilon E\left(\boldsymbol{X}_{p}\right)=\boldsymbol{X}_{d}
$$

$$
E[g(\boldsymbol{X})]=\mathrm{E}\left[\mathrm{g}\left(\boldsymbol{X}_{d}\right)\right]+\varepsilon E\left[g\left(\boldsymbol{X}_{p}\right)\right]=g_{d}(\boldsymbol{X})
$$

Both sides of Eqs.(3) and (4) are evaluated about the variance and third and fourth moments of the random variables, and the state function as follows:

$$
\begin{gathered}
E[g(\boldsymbol{X})]=\mathrm{E}\left[\mathrm{g}\left(\boldsymbol{X}_{d}\right)\right]+\varepsilon E\left[g\left(\boldsymbol{X}_{p}\right)\right]=g_{d}(\boldsymbol{X}) \\
C_{3}(\boldsymbol{X})=E\left\{[\boldsymbol{X}-E(\boldsymbol{X})]^{[3]}\right\}=\varepsilon^{3}\left[\boldsymbol{X}_{p}^{[3]}\right] \\
C_{4}(\boldsymbol{X})=E\left\{[\boldsymbol{X}-E(\boldsymbol{X})]^{[4]}\right\}=\varepsilon^{4}\left[\boldsymbol{X}_{p}^{[4]}\right] \\
V \operatorname{ar}[g(\boldsymbol{X})]=E\left\{[g(\boldsymbol{X})-E g(\boldsymbol{X})]^{[2]}\right\}=\varepsilon^{2} E\left\{\left[g_{p}(\boldsymbol{X})\right]^{[2]}\right\} \\
C_{3}[g(\boldsymbol{X})]=E\left\{[g(\boldsymbol{X})-E g(\boldsymbol{X})]^{[3]}\right\}=\varepsilon^{3} E\left\{\left[g_{p}(\boldsymbol{X})\right]^{[3]}\right\}(11) \\
C_{4}[g(\boldsymbol{X})]=E\left\{[g(\boldsymbol{X})-E g(\boldsymbol{X})]^{[4]}\right\}=\varepsilon^{4} E\left\{\left[g_{p}(\boldsymbol{X})\right]^{[4]}\right\}(12)
\end{gathered}
$$

According to Kronecker algebra, the notation $(\bullet)^{[k]}$ represents the $k$ th order Kronecker power of an arbitrary matrix. i.e., for the arbitrary matrix $P$, its $k$ th Kronecker power can be expressed as $P^{[k]}=P \otimes P^{[k-1]}=P \otimes P \otimes \cdots P$. The symbol $\otimes$ represents the Kronecker product, which is defined as $(A)_{p \times q} \otimes(B)_{s \times t}=\left(a_{i j} B\right)_{p s \times q t}$.

By expanding the state function $g_{p}(X)$ to a first-order approximation in a Taylor series of the vector-valued and matrix-valued functions at a point $E(\boldsymbol{X}) \approx \boldsymbol{X}_{d}$, which is on the failure surface $E\left[g_{p}(\boldsymbol{X})\right]=0$, the expression of $g_{p}(\boldsymbol{X})$ is given by

$$
g_{p}(\boldsymbol{X})=\frac{\partial g_{d}(\boldsymbol{X})}{\partial \boldsymbol{X}^{T}} \boldsymbol{X}_{p}
$$

Substituting Eq.(13) into Eq.(10-12), we obtain

$$
\begin{gathered}
\sigma_{g}^{2}=\operatorname{Var}[g(\boldsymbol{X})]=\left(\frac{\partial g_{d}(\boldsymbol{X})}{\partial \boldsymbol{X}^{T}}\right)^{[2]} \operatorname{Var}[\operatorname{cs}(\boldsymbol{X})] \\
\theta_{g}=C_{3}[g(\boldsymbol{X})]=\left(\frac{\partial g_{d}(\boldsymbol{X})}{\partial \boldsymbol{X}^{T}}\right)^{[3]} C_{3}[\operatorname{cs}(\boldsymbol{X})] \\
\eta_{g}=C_{4}[g(\boldsymbol{X})]=\left(\frac{\partial g_{d}(\boldsymbol{X})}{\partial \boldsymbol{X}^{T}}\right)^{[4]} C_{4}[\operatorname{cs}(\boldsymbol{X})]
\end{gathered}
$$

The probability density function or joint probability density function of the basic random parameters is needed to calculate the structural reliability or failure probability. However, it is difficult in practice to have enough information to determine their distribution types. Even when the probability distribution of the state function is approximated, it is difficult to obtain failure probability by numerical integration.

Thus, the moment-based method is one of the most practical methods for reliability analysis. In the second-moment method, the reliability can be defined as 


$$
R_{S M} \approx \Phi\left(\beta_{S M}\right)
$$

where

$$
\beta_{S M}=\frac{\mu_{g}}{\sigma_{g}}=\frac{E[g(\boldsymbol{X})]}{\sqrt{\operatorname{Var}[g(\boldsymbol{X})]}}
$$

and $\Phi(\bullet)$ is the cumulative stand normal distribution function, $\mu_{g}$ is the mean value of the state function $g(\boldsymbol{X})$.

The Fourth-moment method can be used under the circumstance of the state function being explicit expression and the first four terms of the random parameters being known. The limitation of the Fourth-moment method is that more moments should be known while comparing to the Second-moment method and thus statistical difficulties may occur. However, because of this limitation, the Fourth-moment method has the advantage when dealing with reliability problems with abnormal distribution functions.

Under the circumstance of the first four moments of state function and random variables being given, and the first four moments of the basic random parameters known, the reliability can be obtained by using the fourth-moment method:

$$
R_{F M} \approx \Phi\left(\beta_{F M}\right)
$$

where

$$
\beta_{F M}=\frac{3\left(\alpha_{4 g}-1\right) \beta_{S M}+\alpha_{3 g}\left(\beta_{S M}^{2}-1\right)}{\sqrt{\left(9 \alpha_{4 g}-5 \alpha_{3 g}^{2}-9\right)\left(\alpha_{4 g}-1\right)}}
$$

And $\alpha_{3 g} 、 \alpha_{4 g}$ is the coefficient of skewness and kurtosis,

$$
\alpha_{3 g}=\frac{E\left[\left(\boldsymbol{X}-\mu_{g}\right)^{3}\right]}{\sigma_{g}^{3}}=\frac{\theta_{g}}{\sigma_{g}^{3}} \alpha_{4 g}=\frac{E\left[\left(\boldsymbol{X}-\mu_{g}\right)^{4}\right]}{\sigma_{g}^{4}}=\frac{\eta_{g}}{\sigma_{g}^{4}}
$$

where $\sigma_{g}, \theta_{g}$ and $\eta_{g}$ are obtained from Eqs. (14) to (16), respectively. The computational relationship between the skewness and Kurtosis of arbitrary random variables in the state equations is $\alpha_{4 g} \geq \alpha_{3 g}^{2}+1$.

\section{RELIABILITY SENSITIVITY}

The reliability sensitivity with respect to the mean value $\boldsymbol{X}_{d}$ and variance $\operatorname{Var}(\boldsymbol{X})$ of the basic random parameters $\boldsymbol{X}$ is derived as follows:

$$
\begin{gathered}
\frac{d R_{F M}\left(\beta_{F M}\right)}{d \overline{\boldsymbol{X}}^{T}}=\frac{\partial R\left(\beta_{F M}\right)}{\partial \beta_{F M}} \frac{\partial \beta_{F M}}{\partial \beta_{S M}} \frac{\partial \beta_{S M}}{\partial \mu_{g}} \frac{\partial \mu_{g}}{\partial \overline{\boldsymbol{X}}^{T}} \\
\frac{d R_{F M}}{d \operatorname{Var}(\boldsymbol{X})}=\left[\frac{\partial R_{F M}}{\partial \beta_{F M}}\left(\frac{\partial \beta_{F M}}{\partial \beta_{S M}} \frac{\partial \beta_{S M}}{\partial \sigma_{g}}+\frac{\partial \beta_{F M}}{\partial \sigma_{g}}\right)\right] \frac{\partial \sigma_{g}}{\partial \operatorname{Var}(\boldsymbol{X})}
\end{gathered}
$$

The terms on the right side of Eqs. (22) and (23) can be expressed as

$$
\frac{\partial R_{F M}}{\partial \beta_{F M}}=\varphi\left(\beta_{F M}\right)
$$

where $\varphi(\bullet)$ is the probability density function of standard normal distribution,

$$
\begin{aligned}
& \frac{\partial \beta_{F M}}{\partial \beta_{S M}}=\frac{3\left(\alpha_{4 g}-1\right)+2 \alpha_{3 g} \beta_{S M}}{\sqrt{\left(9 \alpha_{4 g}-5 \alpha_{3 g}^{2}-9\right)\left(\alpha_{4 g}-1\right)}} \\
& \frac{\partial \beta_{S M}}{\partial \mu_{g}}=\frac{1}{\sigma_{g}} \\
& \frac{\partial \mu_{g}}{\partial \overline{\boldsymbol{X}}^{T}}=\left[\frac{\partial \bar{g}}{\partial \boldsymbol{X}_{1}} \frac{\partial \bar{g}}{\partial \boldsymbol{X}_{2}} \cdots \frac{\partial \bar{g}}{\partial \boldsymbol{X}_{n}}\right] \\
& \frac{\partial \beta_{S M}}{\partial \sigma_{g}}=-\frac{\mu_{g}}{\sigma_{g}^{2}} \\
& \frac{\partial \sigma_{g}}{\partial \operatorname{Var}(\boldsymbol{X})}=\frac{1}{2 \sigma_{g}}\left[\frac{\partial \bar{g}}{\partial \boldsymbol{X}} \otimes \frac{\partial \bar{g}}{\partial \boldsymbol{X}}\right] \\
& \frac{\partial \beta_{F M}}{\partial \sigma_{g}}=-\frac{1}{\sigma_{g}}\left\{\frac{12 \alpha_{4 g} \beta_{S M}+3 U}{\sqrt{Q M}}\right. \\
& \left.+\frac{\left[M\left(36 \alpha_{4 g}+30 \alpha_{3 g}^{2}\right)-4 \alpha_{4 g} Q\right]\left(3 M \beta_{S M}+U\right)}{2 \sqrt{Q^{3} M^{3}}}\right\}
\end{aligned}
$$

where $Q=9 \alpha_{4 g}-5 \alpha_{3 g}^{2}-9$

$$
\begin{aligned}
& M=\alpha_{4 g}-1 \\
& U=\alpha_{3 g}\left(\beta_{S M}^{2}-1\right)
\end{aligned}
$$

Substituting Eqs. (14)-(21) and (24)-(30) into Eqs. (22) and (23), one will obtain the reliability sensitivity results, $d R_{F M} / d \boldsymbol{X}_{d}^{T}$ and $d R_{F M} / d \operatorname{Var}(\boldsymbol{X})$.

\section{RELIABILITY-BASED ROBUST DESIGN}

Typically, the reliability-based robust design of tracked vehicle components is a multi-objective optimization procedure, where the objectives are to minimize the weight and structural reliability sensitivity with respect to the nominal value of the design variables. Optimum constraints include the expected structural reliability and geometric parameters. Restrictive random direction method is employed to proceed optimization design. The reliability-based robust design problem may be reduced to a selected problem by the complicated objective method, and the multi-objective optimization model is expressed as

$$
\left\{\begin{array}{c}
\min f(\boldsymbol{X})=\sum_{k=1}^{n} w_{k} f_{k}\left(\boldsymbol{X}_{d}\right) \\
\text { s.t.R }-R_{0} \geq 0, q_{i}\left(\boldsymbol{X}_{d}\right) \geq 0, i=1, \cdots, l
\end{array}\right.
$$

where $R_{0}$ is the desired structural reliability, $w_{k}$ are $k$ th weighting factors. The value of $w_{k}$ is determined by the importance of the sub-objective function respectively. In the paper, $w_{k}$ is given as below: 


$$
\left\{\begin{array}{c}
w_{1}=\frac{f_{k}\left(X^{l}\right)-f_{k}\left(X^{k}\right)}{\left[f_{1}\left(X^{k}\right)-f_{1}\left(X^{l}\right)\right]+\left[f_{2}\left(X^{(k-1)}\right)-f_{2}\left(X^{2}\right)\right]+\cdots+\left[f_{k}\left(X^{l}\right)-f_{k}\left(X^{k}\right)\right]} \\
w_{2}=\frac{f_{k-1}\left(X^{2}\right)-f_{k-1}\left(X^{(k-1)}\right)}{\left[f_{1}\left(X^{k}\right)-f_{1}\left(X^{l}\right)\right]+\left[f_{2}\left(X^{(k-1)}\right)-f_{2}\left(X^{2}\right)\right]+\cdots+\left[f_{k}\left(X^{l}\right)-f_{k}\left(X^{k}\right)\right]} \\
\vdots \\
w_{k}=\frac{f_{1}\left(X^{k}\right)-f_{1}\left(X^{1}\right)}{\left[f_{1}\left(X^{k}\right)-f_{1}\left(X^{l}\right)\right]+\left[f_{2}\left(X^{(k-1)}\right)-f_{2}\left(X^{2}\right)\right]+\cdots+\left[f_{k}\left(X^{l}\right)-f_{k}\left(X^{k}\right)\right]}
\end{array}\right.
$$

where $X^{k}$ is the optimal point of the $k$ th sub-objective function. In the following examples, two objective functions are considered. The function $f_{1}\left(\boldsymbol{X}_{d}\right)$ is the area or volume of the tracked vehicle component, and $f_{2}\left(\boldsymbol{X}_{d}\right)$ is the reliability sensitivity with respect to the nominal value of the design parameters $x=\left(x_{1} x_{2} \cdots x_{m}\right)^{T}$, and $q_{i}\left(X_{d}\right)$ is a problem dependent inequality constraint on shown.

\section{EXAMPLES}

\section{A. Reliability-Based Robust Design of a Capstan}

A capstan (Fig. 1) is an important power transmission component in drive system, it can transfer the power from the drive shaft to track wheel, which engages through the gear and track wheel, it can toggle track campaign, only to withstand the torque effect.

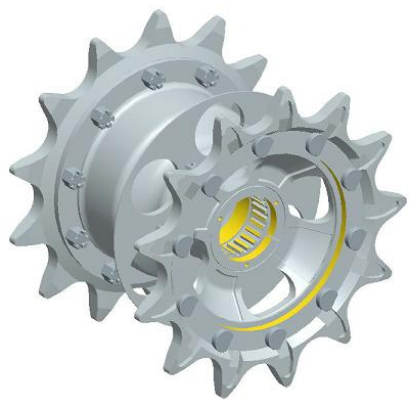

Fig. 1. Solid models of capstan

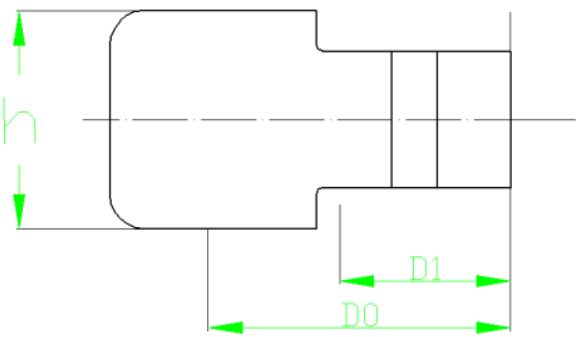

Fig. 2. Structure sketch of a gear on capstan.

The tensile stresses of the capstan is

$$
\tau=\frac{3 P\left(D_{0}-D_{1}\right)}{\pi D_{1} h^{2}}
$$

where $P$ is the tensile load on the capstan, and the cross section dimensions are $h, D_{0}, D_{1}, P$.

From reliability theory, the state equation of the capstan is defined as

$$
g(X)=r-\tau
$$

where $r$ is the material strength. The random variable vector is given by $X=\left(r D_{0} D_{1} h P\right)^{T}$.

1) Generally speaking, dimensions and material properties are usually within normal distribution. The statistical characterization of material strength $r$ is $\left(\mu_{r}, \sigma_{r}\right)=(135,5.256) \mathrm{MPa} \quad$. The dimensions are $\left(\mu_{h}, \sigma_{h}\right)=(50,0.25) \mathrm{mm},\left(\mu_{D_{0}}, \sigma_{D_{0}}\right)=(1200,6) \mathrm{mm}$, $\left(\mu_{D_{1}}, \sigma_{D_{1}}\right)=(1000,5) \mathrm{mm}$. The load $P$ is an arbitrary distributed random variable, with first four moments $\left(\mu_{P}, \sigma_{P}, \theta_{P}, \eta_{P}\right)=\left(1.3025 \times 10^{6} \mathrm{~N}, 1.2024 \times 10^{5} \mathrm{~N}^{2}, 1.9872 \times 10^{15} \mathrm{~N}^{3}, 1.5724 \times 10^{21} \mathrm{~N}^{4}\right)$ Then, the reliability index, the reliability, and the reliability sensitivities can be determined as

$$
\begin{aligned}
& \beta_{S M}=3.102912, \beta_{F M}=2.466713 \\
& R_{F M}=0.9931821, R_{M C S}=0.9935
\end{aligned}
$$

$$
d R / d \overline{\boldsymbol{X}}^{T}=\left[\begin{array}{c}
\partial R / \partial r \\
\partial R / \partial D_{0} \\
\partial R / \partial D_{1} \\
\partial R / \partial h \\
\partial R / \partial P
\end{array}\right]^{T}=\left[\begin{array}{c}
8.096 \times 10^{-4} \\
-5.586 \times 10^{-4} \\
6.737 \times 10^{-4} \\
4.709 \times 10^{-3} \\
-6.703 \times 10^{-8}
\end{array}\right]^{T}
$$

2) If the desired reliability $R_{0}$ equals 0.999 , we can perform the optimization design of capstan dimensions $r, D_{0}, D_{1}, h$ and $P$.

First, we set up the objective function: (a) to minimize the capstan weight by minimizing the section area $f_{1}(x)$; (b) to minimize the reliability sensitivity with respect to the mean value of design variable $\boldsymbol{x}=\left(r, D_{0}, D_{1}, h, P\right)^{T}$.

$$
f_{1}(x)=\frac{\pi}{4}\left(1+x_{1}^{2}\right)\left(x_{2} x_{3}\right)^{3} x_{4} x_{5}
$$

$$
f_{2}(x)=\sqrt{\sum_{i=1}^{5}\left(\frac{\partial R_{F M}}{\partial x_{i}}\right)^{2}}
$$

Second, we set up the optimization constraints:

$$
\begin{gathered}
g-\Phi^{-1}\left(R_{0}\right) \sigma_{g} \geq 0 \\
x_{2}-x_{1}>100.0, x_{2}-2 x_{3}>0,1.4<\frac{x_{4}}{x_{3}}<2.8
\end{gathered}
$$

Third, given initial values $r=135 \mathrm{MPa}$, $D_{0}=1200 \mathrm{~mm} \quad, \quad D_{1}=1000 \mathrm{~mm} \quad, \quad h=50 \mathrm{~mm}$, $P=13025000$ N.

Common reliability optimization method is applied and only the lightest weight of the capstan is considered, that is, single objective function of the minimum area $f_{1}(x)$ need to be resolved. The obtained minimum area and sizes of the capstan are as follows:

$$
S=6447.012 \mathrm{~mm}^{2}, r=138.67 \mathrm{MPa}, D_{0}=1159.62 \mathrm{~mm}
$$




$$
D_{1}=983.64 \mathrm{~mm}, h=53.49 \mathrm{~mm}, P=13.375 \mathrm{MPa}
$$

According to the results of reliability optimization, the reliability index, reliability and reliability sensitivity of the tension bar are calculated out as follows:

$$
\begin{aligned}
& \beta_{S M}= 3.081576, \beta_{F M}=2.432581, \\
& R_{F M}= 0.9919524, R_{M C S}=0.9929 \\
& d R / d \overline{\boldsymbol{X}}^{T}=\left[\begin{array}{c}
\partial R / \partial r \\
\partial R / \partial D_{0} \\
\partial R / \partial D_{1} \\
\partial R / \partial h \\
\partial R / \partial P
\end{array}\right]^{T}=\left[\begin{array}{c}
9.035 \times 10^{-4} \\
-5.978 \times 10^{-4} \\
1.0561 \times 10^{-3} \\
6.192 \times 10^{-3} \\
-4.397 \times 10^{-7}
\end{array}\right]^{T}
\end{aligned}
$$

Fourth, the reliability-based robust optimized results are

$$
\begin{gathered}
S=6512.947 \mathrm{~mm}^{2}, r=136.09 \mathrm{MPa}, D_{0}=1189.36 \mathrm{~mm} \\
D_{1}=1004.37 \mathrm{~mm}, h=49.62 \mathrm{~mm}, P=13.289 \mathrm{MPa}
\end{gathered}
$$

Based on the results computed by the reliability-based robust design approach, the reliability index, the reliability, and the reliability sensitivity of the capstan become

$$
\begin{aligned}
\beta_{S M}= & 3.287312, \beta_{F M}=2.57196, \\
R_{F M}= & 0.9991982, R_{M C S}=0.9962 \\
d R / d \overline{\boldsymbol{X}}^{T}= & {\left[\begin{array}{c}
\partial R / \partial r \\
\partial R / \partial D_{0} \\
\partial R / \partial D_{1} \\
\partial R / \partial h \\
\partial R / \partial P
\end{array}\right]^{T}=\left[\begin{array}{c}
7.896 \times 10^{-4} \\
-3.232 \times 10^{-4} \\
5.532 \times 10^{-4} \\
2.198 \times 10^{-3} \\
-8.562 \times 10^{-9}
\end{array}\right]^{T} }
\end{aligned}
$$

From the results obtained by common reliability optimization method and reliability sensitivity method respectively, it can be found out that the value of reliability sensitivity of the design sizes $h, D_{0}, D_{1}, P$ of the capstan has an obvious decrease after reliability-based robust design. That is, the reliability of the capstan is not sensitive to the variance of the design sizes $h, D_{0}, D_{1}, P$, and the safe reliability and robustness of the capstan have been improved.

\section{B. Reliability-Based Robust Design of a Drive Shaft}

A drive shaft (Fig. 3) is another important power transmission components, it can transfer the power from the side reducer to capstan, which fixed in the capstan though internal splined sleeve, to withstand the torque and moment effect.

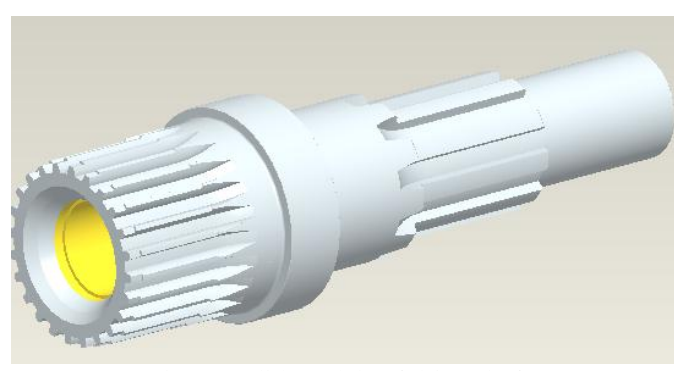

Fig. 3. Solid models of drive shaft.

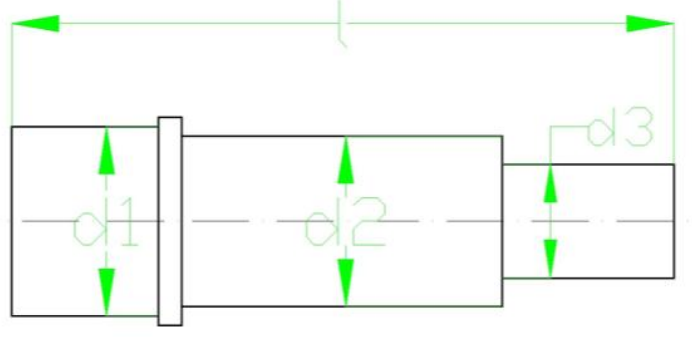

Fig. 4. Structure sketch of a drive shaft.

The mechanical model of drive shaft (Fig. 5), thus, the shear stress and the normal stress on the drive shaft are

$$
\begin{gathered}
\tau=\frac{T}{W_{\rho}} \\
\sigma=\frac{M}{W_{x}}
\end{gathered}
$$

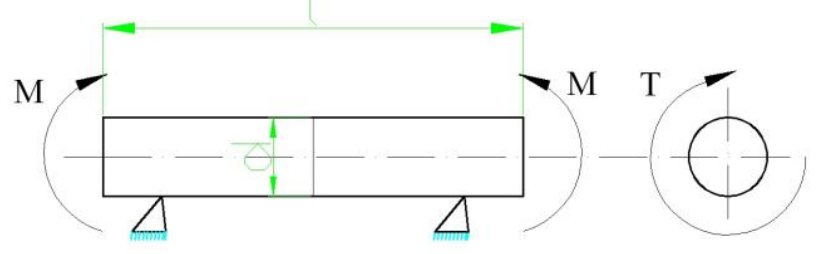

Fig. 5. Mechanical model of drive shaft.

where $W_{\rho}$ is the coefficient in torsion, $W_{x}$ is the coefficient in bending strength. From material mechanics, the coefficient in torsion and the coefficient in bending strength of the drive shaft are defined as

$$
\begin{aligned}
& W_{\rho}=\frac{\pi}{16 D}\left(1-\alpha^{4}\right) \\
& W_{x}=\frac{\pi}{32 D}\left(1-\alpha^{4}\right)
\end{aligned}
$$

where $\alpha=1-(d / D)^{4} ; d$ is the bore of drive shaft, $D$ is the outside diameter of drive shaft.

When the shaft is stepped shaft, the diameter can be replaced with $\quad D=\min \left(D_{1}, D_{2}, \cdots, D_{n}\right) \quad$ and $d=\max \left(d_{1}, d_{2}, \cdots, d_{n}\right)$ for correction.

From material mechanics, the maximum stress of the drive shaft is defined as

$$
\lambda=\sqrt{\sigma^{2}+(\kappa \tau)^{2}}
$$

where $\kappa$ is stress reduced value, general equal to 0.6.

From reliability theory, the state equation of the capstan is defined as

$$
g(X)=r-\sqrt{\sigma^{2}+(\kappa \tau)^{2}}
$$

where $r$ is the material strength. The random variable vector is given by $X=(r d D M T)^{T}$.

1) The statistical characterization of material strength $r$ is $\left(\mu_{r}, \sigma_{r}\right)=(135,5.256) \mathrm{MPa}$. The dimensions of the drive shaft are $\quad D_{1}=(120,6) \mathrm{mm}$ 
$D_{2}=(110,5) \mathrm{mm}, D_{3}=(100,5) \mathrm{mm}$. To minimize the drive shaft weight by replace the shaft with hollow shaft. Give initial value $d=(35,8) \mathrm{mm}$. The load $M$ and $T$ are an arbitrary distributed random variable, with first four moments.

$$
\begin{aligned}
& M=\left(2.5619 \times 10^{3} N, 1.3758 \times 10^{2} N^{2}, 5.3951 \times 10^{10} N^{3},\right. \\
& \left.2.3482 \times 10^{15} N^{4}\right) \\
& T=\left(1.3025 \times 10^{6} N, 1.2024 \times 10^{5} N^{2}, 1.9872 \times 10^{15} N^{3},\right. \\
& \left.1.5724 \times 10^{21} N^{4}\right)
\end{aligned}
$$

Then, the reliability index, the reliability, and the reliability sensitivities can be determined as

$$
\begin{gathered}
\beta_{S M}=3.102912 \beta_{F M}=2.466713 R_{F M}=0.9931821 \\
R_{M C S}=0.9935 \\
d R / d \bar{X}^{T}=\left[\begin{array}{c}
\partial R / \partial r \\
\partial R / \partial d \\
\partial R / \partial D_{3} \\
\partial R / \partial M \\
\partial R / \partial T
\end{array}\right]^{T}=\left[\begin{array}{c}
7.321 \times 10^{-5} \\
-9.754 \times 10^{-5} \\
5.308 \times 10^{-4} \\
-5.731 \times 10^{-7} \\
-6.086 \times 10^{-8}
\end{array}\right]^{T}
\end{gathered}
$$

According to the results obtained by reliability sensitivity, it can be found out that the reliability of drive shaft rises when the mean value of $r$ and $D_{3}$ increase, and the reliability falls when the mean value of $d$ and load increase. Besides, the maximal rate of change is dimension $D_{3}$, and $d, r, M, T$ are in turns. Which means the diameter $D_{3}$ needs to be changed first in the process of design and manufacture when the drive shaft must be more reliable.

2) Because of the drive shaft should be connected with the capstan, the diameter $D_{3}$ can't be changed easily, to minimize the drive shaft weight and the reliability sensitivity with respect to the mean value of design variable $X=(r d D M T)^{T}$ by changing inside diameter $d$. The reliability and quality of drive shaft with different inside diameter $d$ can be determined in Table I.

TABLE I: RELIABILITY AND QUALITY OF DRIVE SHAFT ON DIFFERENT D/D AS FIXED D

\begin{tabular}{cccccc}
\hline \hline $\mathrm{d}(\mathrm{mm})$ & 35 & 42 & 50 & 58 & 62.5 \\
$\mathrm{~d} / \mathrm{D} 3$ & 0.35 & 0.42 & 0.50 & 0.58 & 0.625 \\
Reliability & 0.9932 & 0.9805 & 0.9712 & 0.9506 & 0.9272 \\
Quality (kg) & 34.5 & 32.6 & 29.9 & 26.7 & 25.1 \\
\hline \hline
\end{tabular}

According to the results in Tab I, it can be found out that the reliability of drive shaft falls when the mean value of inside diameter $d$ increase and the quality also decrease. Further more, when the inside diameter $d$ increases $20 \%$, the quality decreases $5 \%$, but the reliability decreases just $1.3 \%$, satisfied the requirement of reliability, so the drive shaft can be replaced with hollow shaft, and the inside diameter $d$ can be designed depend on required reliability.

\section{CONCLUSION}

1) The fourth moment approach for reliability sensitivity design of vehicle components is proposed, an assessment of influence of changes of determined design parameters on vehicle components' reliability is made to fully reflect the sensitivity of each design parameter to vehicle components' failure.

2) This numerical reliability-based robust design technique can improve the design level of vehicle components and reduce the manufacturing cost, and it also provides a theoretical principle for reliability-based optimization design of vehicle components.

3) Compared with common optimization computing method, it is clear that the reliability obtained by using the technique proposed rises obviously $(>0.04 \%)$, and the reliability-based sensitivity's absolute value decreases (reduction $>0.01 \%$ ). Therefore, it is clear that in manufacturing or using, tiny variation of design parameters can fulfill both the safety requirements and the robust requirements.

4) According to the sensitivity analysis results from the drive shaft, the hollow shaft already meets reliability requirements. This will not only reduce the quality of the shaft, it can also be refined according to the reliability of the design requirements.

\section{REFERENCES}

[1] C.-M. Lu, Y.-M. Zhang, Y. Liu et al., "Sensitivity analysis for frequency reliability of a random bending vibration system of continuous beam," Journal of Vibration and Shock, vol. 32, pp. 159-162, April 2013.

[2] S. Adhikari, "Sensitivity based reduced approaches for structural reliability analysis," Sadhana, vol. 35, pp. 319-339, April 2010.

[3] A. V. Marcos and I. S. Gerhart, "A survey on approaches for reliability-based optimization," Structural and Multidisciplinary Optimization, vol. 42, pp. 645-663, May 2010.

[4] Y.-M. Huang, W. Liu, Y.-S. Liu, Z.-F. Yue, "Parameter sensitivity and resonance reliability of a fluid-filled pipeline," Journal of Vibration and Shock, vol. 29, pp. 193-195, January 2010.

[5] J. P. Auzins and A. F. Boyko, "Analysis of the sensitivity of a vibration-based procedure for structural identification," Strength of Materials, vol. 38, pp. 435-441, April 2006.

[6] Y.-M. Liu, X.-L. Zhang, B. Yang, "MATLAB-based four moment method for reliability and optimization design," Mechanical Engineering \& Automation, vol. 22, pp. 27-29, June 2011.

[7] X.-D. He, Y.-M. Zhang, B.-C. Wen, "Stable reliability sensitivity design of compressive bar with non-normal distribution parameters," Journal of Astronautics, vol. 28, pp. 1401-1404, May 2007.

[8] Y.-M. Zhang and Z. Yang, "Reliability-based sensitivity analysis of vehicle components with non-normal distribution parameters," International Journal of Automotive Technology, vol. 10, pp. 181-194, February 2009.

[9] A. Hartwich, K. Stockmann, C. Terboven et al., "Parallel sensitivity analysis for efficient large-scale dynamic optimization," Optimization and Engineering, vol. 12, pp. 489-508, April 2011.

[10] H. Xu and S. Rahman, "Decomposition methods for structural reliability analysis," Probabilistic Engineering Mechanics, vol. 20, pp. 239-250, July 2005.

[11] S. Rahman and D. Wei, "Design sensitivity and reliability-based structural optimization by univariate decomposition," Structural and Multidisciplinary Optimization, vol. 35, pp. 245-261, March 2008.

[12] J. Song and Z.-Z. Lu, "A new reliability sensitivity analysis method," Acta Aeronautica Et Astronautica Sinica, vol. 27, pp. 823-826, May 2006. 
[13] K. P. Kim and H. Huh, "Dynamic limit analysis formulation for impact simulation of structural members," Int. J. Solids \& Struc, vol. 43, pp. 6488-6501, October 2006.

[14] R. H. Sues and M. A. Cesare, "System reliability and sensitivity factors via the MPPSS method," Probab. Eng. Mech, vol. 20, pp. 148-157, February 2005

[15] L. Gu and R. J. Yang, "On reliability-based optimisation methods for automotive structures," Int. J. Mater. \& Prod. Technol, vol. 25, pp. 3-26, January 2006.

[16] B. A. Tolson, H. R. Maier, A. R. Simpson et al., "Genetic algorithms for reliability-based optimization of water distribution systems," $J$ Water Resour. Plann. Manage, vol. 130, pp. 63-72, January 2004.

[17] B. D. Youn, K. K. Choi, R. J. Yang et al., "Reliability-based design optimization for crashworthiness of vehicle side impact," Struct. Multidiscip. Opt., vol. 26, pp. 272-283, March 2004.

[18] C. Zang, M. I. Friswell, and J. E. Mottershead, "A review of robust optimal design and its application in dynamics," Comput. Struct., vol. 83, pp. 315-326, April 2005.

[19] R. E. Melchers and M. Ahammed, "A fast approximate method for parameter sensitivity estimation in Monte Carlo structural reliability," Comput. Struct., vol. 81, pp. 55-61, January 2004.
[20] S. Gunawan and S. Azarm, "Multi-objective robust optimization using a sensitivity region concept," Strbct. Multidiscip. Opt., vol. 29, pp. 50-60, January 2005.

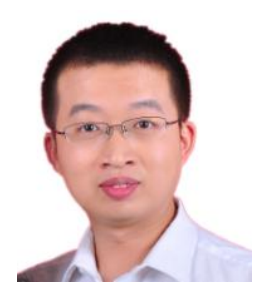

Zhe Liu was born in Shanxi, P. R. China in 1988. $\mathrm{He}$ is a $\mathrm{PhD}$ graduate student at the First Department, Mechanical Engineering College, China. He received his master's degree from Mechanical Engineering College in 2013. His research interests include mechanical engineering systems life prediction, computer simulation reliability. He is the author or coauthor of more than 10 scientific papers.

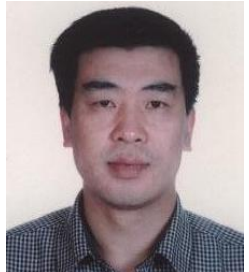

Fenghe Tao was born in 1963. He is a professor at the First Department, Mechanical Engineering College, China. He received his Ph.D. from the Six Department, Mechanical Engineering College in 2007. His currently research include mechanical engineering systems reliability analysis and architecture optimization. $\mathrm{He}$ is the author or coauthor of more than 60 scientific papers. 